\title{
EDITORIAL
}

\section{Recruitment to psychiatry: a global problem}

\author{
Tom Brown ${ }^{1}$ and Howard Ryland ${ }^{2}$
}

${ }^{1}$ Retired Consultant Psychiatrist, Western Infirmary Glasgow, G11 6NT, UK; Former Royal College of Psychiatrists Associate Registrar for Recruitment (201116); email tmb0655@yahoo.com

${ }^{2}$ South West London and St George's Mental Health National Health Service Trust, London, UK

Conflicts of interest. Howard Ryland was an officer of the European Federation of

Psychiatric Trainees.

doi:10.1192/bji.2017.29

(c) The Authors 2018. This is an Open Access article, distributed under the terms of the Creative Commons Attribution-

NonCommercial-NoDerivatives licence (http://creativecommo mons. org/licenses/by-nc-nd/4.0/), which permits non-commercial re-use, distribution, and reproduction in any medium, provided the original work is unaltered and is properly cited. The written permission of Cambridge University Prission of Cambridge University Press must be obtained for commercial re-use or in order to create a derivative work.
Recruitment to psychiatry is widely recognised to be a global problem, with the World Psychiatric Association (WPA) stating that there are few countries in which it is not an issue (Shields et al, 2016). It is clear that the extent of this problem is hugely variable. Most of Europe (including the UK, where the authors work), the USA, Australia, New Zealand and Japan comfortably exceed the WPA target of 1 psychiatrist per 10000 of the population, whereas in Africa and South East Asia rates are as low as 0.2 per 10000 , with some countries having no psychiatrists at all.

This editorial highlights some key themes in the international literature over the past few years and highlights some proposed suggestions for addressing the recruitment problem.

In the UK, where the Royal College of Psychiatrists has had a recruitment campaign since 2012 (Royal College of Psychiatrists, 2012), the number of consultant psychiatrists has increased by over $50 \%$ since 2000 (Davies, 2013). However, there is still a shortfall in terms of recruitment despite a small increase in the absolute numbers recruited (perhaps a better measure of the success of recruitment initiatives than so-called 'fill rates' to training posts). Goldacre et al (2013), in the most extensive review to date of recruitment to psychiatry in the UK, note that medical students' views of psychiatry are more polarised than their views on other specialties. They also note that, despite a variety of initiatives, the percentage of medical graduates choosing psychiatry as a career has changed little in 35 years, remaining at a steady $4-5 \%$. Similar problems have been identified in other parts of the world including the USA, Australia and New Zealand. This situation often results in doctors from lower-income countries moving to higherincome countries to 'fill the gaps', leaving the already poorly staffed services in their native countries in an even worse state.

\section{Push and pull factors}

Studies have looked at both 'pull' factors - that is, what attracts people to psychiatry (Farooq et al, 2013) - and so-called 'push' factors (Malhi et al, 2011) that mitigate against the choice of psychiatry as a career. The former include gender (traditionally, more women than men have chosen psychiatry), aspects of personality, exposure to mental illness, teaching, and the length and quality of clinical placements. The latter include the perception that the specialty is unscientific and remote from medicine, that patients with mental illness are untreatable and that psychiatry enjoys low prestige within the medical profession. Indeed, stigma - within the profession and in society at large - looms large in this literature. One shortcoming of the literature is that more than half of it is from the USA and the UK, and there is very little from Africa, Asia or South America. One study that has reviewed the problems of lower-income countries is that by Nortje \& Seedat (2013). They highlight the fact that psychiatry's recruitment problems affect lowand middle-income countries disproportionately. Major reasons for this include stigma (which is perhaps even worse in these countries), the status of psychiatry, lack of trainers, local cultural beliefs, poor working conditions and quality of patient care. They propose a variety of means for addressing these problems, including campaigns to tackle stigma, visits from international teachers, improving undergraduate teaching, more support for the small number of local psychiatrists, and improving infrastructure and services. In many low- and middle-income countries, and indeed in remote and rural areas of other countries, a different model of care may be more realistic and indeed appropriate, with a smaller number of psychiatrists supporting and supervising other health professionals in delivering services.

One potential pull factor that may have been underemphasised in the literature is the correlation between physical and mental health. This, together with the need for integration between physical and mental health services (Prince et al, 2007), cannot be overemphasised. Highlighting this issue to medical students and recently qualified doctors may enhance recruitment to psychiatry.

\section{The views of international psychiatric associations}

The WPA has identified similar push and pull factors to those outlined above. In the WPA's view, medical students' experience of psychiatry is of fundamental importance, and there is a recognition that this varies considerably throughout the world. In countries where psychiatry is predominantly limited to in-patient facilities, where conditions may be poor, the undergraduate experience of psychiatry is more likely to be a negative one. To improve recruitment may therefore require a complete transformation of the type and availability of care for people with mental illness. Conversely, a positive experience of a psychiatry rotation, 
supported by undergraduate exposure to psychology, social sciences, psychotherapy and 'extracurricular enrichment opportunities' such as electives, psychiatry societies and special study modules, may all encourage students to choose psychiatry.

The WPA has proposed that issues with recruitment to psychiatry and the stigmatisation of the profession and psychiatry more generally are inextricably interlinked. This contention is supported in the wider literature. In 2010, the WPA published guidance on how to combat stigmatisation of both psychiatry and psychiatrists (Sartorius et al, 2010), identifying negative perceptions of psychiatrists among the public, medical students, other health professionals, patients and relatives, and the media. The authors identified interventions from the published literature to combat stigma, focusing predominantly on positive engagement with the media. The guidance recommends that national psychiatric associations work more closely with the media and with other professional and patient organisations, to improve psychiatry training at undergraduate and postgraduate levels and to set and enhance standards of psychiatric care. In its position statement on recruitment in 2016 (Shields et al, 2016), the WPA makes a number of recommendations about improving recruitment in psychiatry. These include introducing measures to improve services in low- and middle-income countries, increasing the quality and quantity of undergraduate teaching, reducing stigma, influencing medical student selection, and encouraging 'enrichment activities' such as those described earlier.

The European Psychiatric Association (EPA) has developed its own guidance on improving the image of psychiatry. The association recognises that stigma against psychiatrists and mental health professionals in general is not widely discussed, but affects recruitment into psychiatry (Bhugra et al, 2015). The EPA guidance goes on to recommend a number of measures to improve the image of psychiatry, including building self-esteem and selfconfidence, stopping self-stigma, integrating mental and physical health, using the biopsychosocial model as an integrative framework and more effective self-marketing. With regard to the recruitment of medical students specifically, the EPA advocates earlier contact with psychiatry, strengthening links with neuroscience and a focus on liaison psychiatry. The effectiveness of anti-stigma initiatives for medical students is questioned, and there is a recognition that there must be support for psychiatry from across medical faculties, to counter the perception that psychiatrists are poor role models. This position is echoed by the European Federation of Psychiatric Trainees (EFPT), whose statement on recruitment recognises that promoting a positive image of psychiatry is essential to supporting recruitment. The EFPT also recognises that psychiatrists in training have a vital role to play in this through their contact with medical students (EFPT, 2016).

\section{Conclusions and recommendations}

Problems recruiting medical students to psychiatry are a global phenomenon. Many of the factors that have been identified as underlying this issue are same worldwide, even if it is acknowledged that some of them, such as stigma, poorquality services, poor working conditions and low income compared with other doctors, disproportionately affect low- and middle-income countries. There is reasonable consensus about what should be done to combat the problem, especially around tackling stigma and improving undergraduate teaching of medical students. As well as dealing with stigma in society at large, stigma within the medical profession needs to be highlighted and tackled. With regard to undergraduate teaching, there is broad agreement that exposure to psychiatry early in the curriculum and repeated exposures throughout medical school are crucial, as indeed is the quality of teaching (in this respect, undergraduate teaching can be either a 'push' factor or a 'pull' factor). We believe that undergraduate exposure to psychiatry could involve greater use of recovered patients in the teaching of students, as often placements are too short for students to experience the very real recovery that patients can make exacerbating beliefs about the 'untreatability' of such patients.

Most countries that have active recruitment campaigns have focused their activities primarily on medical students and recently qualified doctors, though some also have a focus on senior school students considering a medical career or even a career in psychology (Royal College of Psychiatrists, 2012). Much more could be done to share innovative initiatives to tackle recruitment between countries. International professional organisations could help to support such an exchange of best practice.

Psychiatrists have sometimes been their own worst enemies with regard to recruitment. The frequent and often sterile debates we have about whether biomedical or psychological approaches to our specialty are best is a case in point. In reality, both approaches are required. The psychiatrist of the future will need to be well grounded in neuroscience but also to have a solid understanding of the importance of psychological and social factors in the aetiology and treatment of psychiatric disorders. In our obsession with the number of psychiatrists we need, we must not lose sight of their quality. Davies (2013) has wisely cautioned that a desire to increase numbers at all costs may be 'perceived as an attempt to maintain a diffuse jack-of-all-trades special interest group rather than specialty'. This is a pitfall we must surely avoid. Moreover, having an adequate number of psychiatrists does not guarantee high-quality services, and other factors, such as strengthening human resources in general and respect for human rights, may be equally or even more important. 
In conclusion, in an editorial published in 2012 (Lancet, 2012) the author wrote of psychiatry: 'it is time for the specialty to stop devaluing itself because of its chequered history of mental asylums and pseudo-science, and to realign itself as a key biomedical specialty at the heart of mental health'. We would heartily endorse this.

\section{References}

Bhugra D., Sartorius N., Fiorillo A., et al (2015) EPA guidance on how to improve the image of psychiatry and of the psychiatrist. European Psychaitry, 30, 423-430.

Davies T. (2013) Recruitment into psychiatry: quantitative myths and qualitative challenges. British Journal of Psychiatry, 202, 163-165.

European Federation of Psychiatric Trainees (2016) Statements of the European Federation of Psychiatric Trainees (EFP 2014-2016). Available at http://efpt.eu/training/statement/.

Farooq K., Lydall G. J. \& Bhugra D. (2013) What attracts medical students to psychiatry? A review of factors before and during medical school. International Review of Psychiatry, 25, 371-377.

Goldacre M. J., Fazal S., Smith F., et al (2013) Choice and rejection of psychiatry as a career: survey of UK medical graduates from 1974 to 2009. British Journal of Psychiatry, 202, 228-234.
Lancet (2012) Editorial: Psychiatry's identity crisis. Lancet, 379, 1274.

Malhi G. S., Coulston C. M., Parker G. B., et al (2011) Who picks psychiatry? Perceptions, preferences and personality of medical students. Australian and New Zealand Journal of Psychiatry, 45, $861-870$

Nortje G. \& Seedat S. (2013) Recruiting medical students into psychiatry in lower income countries. International Review of Psychiatry, 25, 385-398.

Prince M., Patel V., Shekhar S., et al (2007) No health without mental health. Lancet, 370, 859-877.

Royal College of Psychiatrists (2012) Recruitment Strategy 20112016. http://www.rcpsych.ac.uk/pdf/Recruitment\%20Strategy\%20-\% 2010092013.pdf.

Sartorius N., Gaebel W., Cleveland H.-R., et al (2010) WPA guidance on how to combat stigmatization of psychiatry and psychiatrists. World Psychiatry, 9, 131-144.

Shields G., Ng R., Ventriglio A., et al (2016) WPA Position Statement Recruitment in Psychiatry. World Psychiatric Association. http://www. wpanet.org/detail.php?section_id=7\&content_id=1808.

\title{
EDITORIAL
}

\section{Mental health services in Eastern Europe: past, present and future}

\author{
David Skuse $\odot$
}

Professor of Behavioural and Brain Sciences, Population, Policy and Practice, UCL Great Ormond Street Institute of Child Health, 30 Guilford Street, London WC1N 1EH, UK. Email: d.skuse@ucl.ac.uk

\section{doi:10.1192/bji.2018.30}

(C) The Author 2019. This is an Open Access article, distributed under the terms of the Creative Commons Attribution-

NonCommercial-NoDerivatives licence (http://reativecomives commons. org/licenses/by-nc-nd/4.0/), which permits non-commercial re-use, distribution, and reproduction in any medium, provided the original work is unaltered and is properly cited. The written permission of Cambridge Universty mission of Cambridge University Press must be obtained for com mercial re-use or in order to create a derivative work.
Since the collapse of the Soviet system at the end of the 1980s, Eastern European countries that were formerly part of the Soviet bloc have been looking westward for inspiration when considering reform to their mental health services. We consider how those reforms are progressing in three themed articles. Matt Muijen and Andrew McCulloch discuss the effects of a policy decision, made in 2005 by a Europe-wide consortium, to move mental healthcare from an asylum-based model into the community. It is gratifying to hear how many countries have been successful in so doing, but efforts at reform have met with a triple barrier. First, persisting stigma at all levels of society about people with mental illness; second, the relatively high costs of providing comprehensive community outreach services in countries with limited healthcare resources; third, the lack of trained staff at all levels, from psychiatrists to social workers. Onrej Pec provides an overview of how mental health reforms are progressing in the Czech Republic, a country with a reasonably high income in international terms, but which spends less on mental (compared with physical) health services than many others in the EU15. Most psychiatric services are still based in large asylums, containing 500 beds or more. Community services are not yet well developed, in part because of resistance fostered by social stigma. This disappointing news contrasts with the remarkable success of deinstitutionalisation in Bosnia and Herzegovina, according to a report from Enrichetta Placella, a health advisor to the Swiss Agency for Development and Cooperation. She describes the considerable investment made by several Swiss cantons to help their neighbours modernise the provision of psychiatric care. Switzerland has instigated changes to the relevant legal framework and introduced procedures to ensure high standards of training and support to staff. Other initiatives have included national campaigns aimed at reducing stigma and raising awareness of depression and anxiety. Support for patient associations has been key to the successful reform of mental health services, here and elsewhere. Ms Placella emphasises the importance of establishing a process by which novel methods of service delivery can be managed and sustained, by encouraging a 'culture of quality'. The process adopted for the reform of mental health services by the Swiss Agency is a model that could and should be applied in other Eastern European countries that aspire to provide better care to their populations affected by mental disorders. 